\title{
EVOLUCION DEL ESTADO NUTRICIONAL DE EMBARAZADAS ATENDIDAS EN LA RED BASICA DE SALUD, SANTO ANDRE, BRASIL
}

\author{
Elizabeth Fujimori ${ }^{1}$ \\ Luz Marina Núñez de Cassana² \\ Sophia Cornbluth Szarfarc ${ }^{3}$ \\ Ida Maria Vianna de Oliveira ${ }^{4}$ \\ Elvira Maria Guerra-Shinohara ${ }^{5}$
}

Fujimori E, Cassana LMN, Szarfarc SC, Oliveira IMV, Guerra-Shinohara EM. Evolución del estado nutricional de embarazadas atendidas en la red basica de salud, Santo André, Brasil. Rev Latino-am Enfermagem 2001 maio; 9(3):64-9.

El estado nutricional pregestacional y durante el embarazo fue evaluado en 372 gestantes, a través del indice de masa corporal y la Gráfica de Rosso, prospectivamente. Mitad de las mujeres presentó peso pregestacional normal, aún asi 17,7\% tenía bajo peso y 31,3\% sobrepeso. En el tercer trimestre, 18,8\% tenía bajo peso y 28,2\% sobrepeso. El peso al nacer aumentó conforme la gestante fue nutricionalmente mejor natada. Estos resultados señalan que un control antropométrico adecuado posibilita monitorear nutricionalmente las embarazadas, preveniendo y/o controlando la ocurrencia de condiciones materno-fetales indeseables, hecho que reafirma la importancia de la ejecución de esta actividad como rutina en todo el control prenatal.

TERMINOS CLAVES: embarazadas, evaluación nutricional

\section{DEVELOPMENT OF THE NUTRITIONAL CONDITION OF PREGNANT WOMEN ASSISTED BY THE BASIC HEALTH NETWORK, SANTO ANDRÉ, BRAZIL}

Nutritional conditions in the pre-gestacional period and during pregnancy were evaluated in 372 pregnant women based on the body mass index and Rosso's Graph. Approximately half (51\%) the pregnant women presented normal pre-gestacional weight; however, $17.7 \%$ were underweight and $31.3 \%$ overweight. In the third trimester, $18.8 \%$ were underweight and $28.2 \%$ overweight. The weight at birth increased as nutritional conditions during pregnancy improved. These results show the importance of appropriate anthropometric control in order to prevent a bad neonatal prognosis and reaffirms the importance of such activity during the whole routine of pre-natal control.

KEY WORDS: pregnancy, nutritional conditions

\section{EVOLUÇÃO DO ESTADO NUTRICIONAL DE GRÁVIDAS ATENDIDAS NA REDE BÁSICA DE SAÚDE, SANTO ANDRÉ, BRASIL}

O estado nutricional antes e durante a gestação foi avaliado prospectivamente em 372 gestantes através do índice de massa corporal e ou gráfico de Rosso. Metade das mulheres apresentou peso pré-gestacional normal, porém 17,7\% estava abaixo e 31,3\% acima do peso normal. No terceiro trimestre, 18,8\% tinha peso abaixo e 28,2\% acima do normal. 0 peso ao nascer aumentou de acordo com 0 melhor provimento nutricional da gestante. Estes resultados indicam que um controle antropométrico adequado permite monitorar nutricialmente as gestantes, prevenindo e/ou controlando a ocorrência de condições materno-fetais indesejáveis, fato que reafirma a importância da execução desta atividade como rotina no controle pré-natal.

UNITERMOS: grávidas, avaliação nutricional

${ }^{1}$ Profesor Doctor; ${ }^{4}$ Profesor Asociado. Departamento de Enfermería en Salud Colectiva, Escuela de Enfermería, Universidad de São Paulo. Dirección: Av. Dr. Enéas de Carvalho Aguiar, 419 - 05403-000 - São Paulo - São Paulo - Brasil

${ }^{2}$ Profesor Doctor de Enfermería, Faculdad de Ciencias de la Salud, Universidad Nacional de Cajamarca, Perú

${ }^{3}$ Profesor Asociado del Departamento de Nutrición, Faculdad de Salud Pública, Universidad de São Paulo, Brasil

${ }^{5}$ Doctor Investigador de la Sección de Hematología del Instituto de Adolfo Lutz, Secretaria de Salud del Estado de São Paulo 
INTRODUCCIÓN

$\boldsymbol{E}_{\text {s conocido que el estado nutricional de la gestante, antes }}$ y durante el embarazo es factor fundamental para la salud de ella misma y de su hijo, situación importante a ser considerada, una vez que estas mujeres constituyen un grupo nutricionalmente vulnerable, especialmente en los países en desarrollo ${ }^{(1)}$.

La relación entre desnutrición materna y bajo peso al nacer es tan clara que ya en la década del 60 era utilizada la proporción de recién nacidos de bajo peso como un indicador del estado nutricional de la población gestante ${ }^{(2)}$. Es así que desde el punto de vista de salud pública, el peso al nacer es el parámetro que se relaciona más estrechamente con la supervivencia, con el crecimiento antropométrico y con el desarrollo mental del recién nacido.

Por lo tanto, el diagnóstico precoz del estado nutricional de las embarazadas, la recuperación nutricional manifestada por un progreso y el adecuado incremento de peso durante este proceso pode reducir considerablemente el riesgo de recién nacidos con peso bajo o insuficiente ${ }^{(3-7)}$.

Dos indicadores del estado nutricional materno mostraron asociaciones compatibles con el peso al nacer. Son ellos, el tamaño corporal pregestacional (el peso ${ }^{(8)}$ y estatura ${ }^{(9)}$ de la madre) y el aumento de peso materno durante el embarazo ${ }^{(10)}$.

Siendo el control de los indicadores antropométricos, útil para tamizar mujeres a riesgo nutricional y predecir resultados adversos tanto para la madre como para el niño, el Programa de Atención Integral a la Salud de la Mujer del Ministério de Salud del Brasil incluye como una de las actividades de la asistencia prenatal, la evaluación del estado nutricional a través del índice de peso para estatura para edad gestacional, operacionalizada con la utilización de la gráfica de Rosso ${ }^{(11)}$.

A pesar de esa formalización, la evaluación nutricional de la gestante no es realizada rutinariamente en la atención primaria de salud, limitando el monitoreo y como consecuencia la prevención de riesgos de resultados adversos para el binómio madre-niño.

Así, considerando que la nutrición de la mujer, antes y durante el embarazo es crítica para la salud y supervivencia de ella y de su hijo y que, la evaluación del estado nutricional materno es esencial para identificar mujeres con riesgo de complicaciones gestacionales y de dar a luz infantes con retraso del crecimiento intrauterino, este trabajo tuvo como OBJETIVO evaluar la evolución del estado nutricional de embarazadas que realizaron el prenatal en Puestos de Atención Básica de Salud de la Fundación de Asistencia a la Infancia de Santo André (FAISA), con la finalidad de resaltar la importancia de éste monitoreo.

\section{MATERIAL Y METODOS}

El presente estudio, de naturaleza prospectiva, fue desarrollado en Santo André, ciudad de la Región Metropolitana de São Paulo, Brasil.

La muestra estuvo conformada por 372 embarazadas que iniciaron su control pre-natal en el Programa de Asistencia Integral a la Salud de la Mujer, en uno de los 14 Puestos de Salud de la FAISA, con edad gestacional inferior a 20 semanas al momento de la $1^{\text {a }}$ consulta prenatal y sin patologias asociadas a la gestación.

Los datos socio-económicos y biológicos de las mujeres fueron tomados de las fichas individuales de salud existentes en los servicios. Se consideró edad, ingreso familiar, escolaridad, trabajo fuera del hogar, presencia del compañero, y orden de la gravidez.

El estado nutricional fue evaluado a través de medidas antropométricas (peso y estatura), tomadas con un mínimo de ropa y sin calzado, usando una balanza antropométrica de marca Filizola. El peso fue verificado en cada control prenatal, acompañando la edad gestacional en semanas, cumplidas a partir de la fecha de la última regla menstrual. Como peso pregestacional se utilizó el peso de las mujeres antes de la $10^{\mathrm{a}}$ semana gestacional, dato obtenido solamente para 64 gestantes $(17,2 \%$ ) y la medida de la estatura se obtuvo para 285 mujeres $(76,6 \%)$.

El estado nutricional pregestacional (antes de la $10^{\mathrm{a}}$ semana) fue determinado a través el Indice de Masa Corporal (IMC) utilizándose como puntos de corte los límites propuestos por $^{(12-13)}$ y recomendados por $^{(14)}$.

Para la evaluación del estado nutricional durante el embarazo, la gráfica de incremento de peso para embarazadas ${ }^{(15)}$, validada para la población nacional ${ }^{(16)}$ fue henchida con las medidas de peso y estatura. Esta gráfica clasifica el estado nutricional en tres categorías, según sea la adecuación de la relación peso/estatura de la mujer en cada semana gestacional. La categoría normal es el área comprendida en su límite inferior en la línea que une el peso equivalente al $95 \%$ del estándar en la semana 10 al $122 \%$ en la semana 40 , y en su límite superior la línea que une el peso $115 \%$ en la semana 10 con el peso $131 \%$ en la semana 40 . Las categorías de bajo peso y sobrepeso, fueron aquellas ubicadas por debajo y por encima de los límites. Se ubicó en su estado nutricional a la gestante que tenia verificado edad gestacional mayor a 10 semanas y estatura entre 1,40 a 1,70m. Los resultados fueron analizados en forma transversal, incluyendo el total de las gestantes y en forma longitudinal, involucrando 117 gestantes que tenían las medidas de peso corporal en los tres trimestres.

Los datos fueron pre-codificados y almacenados en un 
único archivo, utilizándose para ello el programa Excell en un computador IBM-PC compatible. Después de verificar la consistencia de las informaciones, éstas fueron analizadas utilizándose el software Epi-Info 6.

\section{RESULTADOS Y DISCUSION}

Entre las características más importantes de las embarazadas, debe señalarse promedio de edad 25,3 \pm 6,1 años, con variación de 14 a 42 años como edad mínima y máxima respectivamente, siendo $21,6 \%$ adolescentes ( $<20$ años). El promedio de edad gestacional en el primer trimestre fue de 10,6 \pm 2,1 semanas, en el segundo trimestre $20,4 \pm 4,0$ y en tercer trimestre 34,0 $\pm 3,6$ semanas. De las que informaron la renta familiar, $38,3 \%$ recibian menos que un salario mínimo vital (US\$ 108,60). Cabe destacar que $62,9 \%$ de las embarazadas no llegaron a culminar su instrucción básica, presentando menos que 8 años de estudio. La mayoría de las mujeres informaron tener compañero $(78,4 \%)$ y no trabajar fuera del hogar $(80,7 \%)$.

En la Tabla 1 se presentan estatura, peso corporal e Indice de Masa Corporal (IMC) pregestacional. Es necesario enfatizar que, no obstante las embarazadas estudiadas tuviesen un control prenatal especial y hiciesen parte del Programa de Atención Integral a la Salud de la Mujer, la medida de la estatura no era verificada de rutina. Esa medida antropométrica, considerada un indicador de la historia nutricional de la madre, refleja la desnutrición crónica y ha sido asociada con el riesgo del bajo peso al nacer ${ }^{(17-19)}$. En el presente estudio se verificó que la proporción de gestantes con estatura inferior a 1,50m, de mayor riesgo obstétrico, fue similar a los $10,8 \%$ observados en estudio epidemiológico desarrollado en Pelotas, RS, en la década del $80^{(18)}$. Teniendo en cuenta la tendencia secular del crescimiento ${ }^{(20)}$, esa proporción debería ser menor, así como encontrado en estudio similar ${ }^{(19)}$, repetido en Pelotas una década después, que verificó disminución de la proporción de mujeres con esa estatura para 4,6\%. En lo que respecta al peso pregestacional este fue analizado solamente para aquellas mujeres que ingresaron al estudio antes de la $10^{\mathrm{a}}$ semana gestacional $(7,6 \pm 1,2)$, considerando que el incremento de peso hasta este período es practicamente nulo. Como se demostró en algunos estudios el peso pregestacional inferior a 50,0Kg también presenta una fuerte asociación con la ocurrencia de bajo peso al nascer ${ }^{(18,}$ 21). En el presente estudio la proporción de gestantes con esa situación nutricional de riesgo $(10,9 \%)$ fue menor que aquellas verificadas en las décadas del 70 (29\%) y del 80 (27,9\%), en las ciudades de São Paulo y Pelotas, respectivamente, como también a los $15,7 \%$, registrados en Pelotas, $\mathrm{RS}$, en la década del $90^{(18-19,22)}$.
Tabla 1 - Constitución corporal y estado nutricional pregestacional. Santo André, 1997

\begin{tabular}{|c|c|c|}
\hline $\begin{array}{c}\text { Constitución corporal } \\
\text { pregestacional }\end{array}$ & $\begin{array}{c}\text { Frecuencia } \\
n(\$)\end{array}$ & Medidas de posición y dispersió \\
\hline \multicolumn{3}{|l|}{ Estatura (m) } \\
\hline$<1,50$ & $28(9,8)$ & Promedio $\pm d s: 1,57 \pm 0,07$ \\
\hline $1,50 \mid--1,55$ & $57(20,0)$ & Mediana: 1,57 \\
\hline $1,55 \mid--1,60$ & $94(33,0)$ & Mínimo: 1,37 \\
\hline $1,60 \mid--1,65$ & $65(22,8)$ & Máximo: 1,75 \\
\hline$\geq 1,65$ & $41(14,4)$ & \\
\hline \multicolumn{3}{|l|}{ Peso (Kg) } \\
\hline$<50,0$ & $7(10,9)$ & Promedio $\pm d s: 60,3 \pm 10,6$ \\
\hline $50,0 \mid--55,0$ & $14(21,9)$ & Mediana: 58,0 \\
\hline $55,0 \mid--60,0$ & $15(23,4)$ & Mínimo: 42,0 \\
\hline$\geq 60,0$ & $28(43,8)$ & Máximo: 91,0 \\
\hline \multicolumn{3}{|l|}{$\begin{array}{l}\text { Indice de masa } \\
\text { corporal }\left(\mathrm{kg} / \mathrm{m}^{2}\right)\end{array}$} \\
\hline Bajo peso & $9(17,7)$ & Promedio $\pm d s: 23,5 \pm 4,2$ \\
\hline Peso ideal & $26(51,0)$ & Mediana: 22,5 \\
\hline Sobrepeso & $12(23,5)$ & Mínimo: 17,7 \\
\hline Obesidade & $4(7,8)$ & Máximo: 35,4 \\
\hline
\end{tabular}

Estudiando la relación entre bajo peso materno y bajo peso al nacer en gestantes atendidas en un Centro de Salud en el contorno de Botucatu, SP, se verificó que, independientemente del estado nutricional de las madres, aquellas con estatura inferior a $155 \mathrm{~cm}$ tuvieron hijos con peso al nacer inferior, comparadas a las de mayor estatura ${ }^{(23)}$. El mismo resultado fue obtenido en el presente estudio, endonde se verificó diferencia estadísticamente significativa $(P=0,01)$ de los promedios de peso al nacer entre mujeres con estatura inferior $(2.981,7 \pm 449,6 \mathrm{~g})$ y superior $(3.270,6 \pm 582,8 \mathrm{~g})$ a $155 \mathrm{~cm}$, independiente del estado nutricional.

EI IMC, calculado para las mujeres que tenían estatura y peso corporal antes de la $10^{a}$ semana gestacional, reveló que solamente la mitad de ellas tenían peso pregestacional ideal. La proporción de gestantes con bajo peso pregestacional fue similar a los $16,8 \%$, obtenidos para la población total de mujeres brasileñas adultas por la Pesquisa Nacional de Salud y Nutrición, 1989 ${ }^{(24)}$. Por otro lado, el exceso de peso (sobrepeso y obesidad) afectaba a casi un tercio de las mujeres estudiadas $(31,3 \%)$, proporción esperada, si se consideran las elevadas prevalencias reportadas (33,8\% a 42,3\%) para las diferentes regiones del país, por la misma Pesquisa Nacional. Sin embargo, comparándose los datos de este estudio con los resultados de investigaciones anteriores, se verificó menor proporción de gestantes com bajo peso, en relación a los relatados para embarazadas adolescentes $(25,2 \%$ ) y de bajo ingreso económico $(29,6 \%)^{(25-26)}$.

Utilizando la relación del peso y estatura para edad gestacional, "curva de incremento de peso para la gestante" ${ }^{(15)}$, se analizó el estado nutricional de la misma embarazada, en tres 
momentos del proceso gestacional, para aquellas mujeres que tenían por lo menos el peso corporal en cada trimestre gestacional $(n=117)$ - estudio longitudinal (Tabla 2) y también el estado nutricional en cinco momentos de la gestación, para la totalidad de las mujeres estudiadas - análisis transversal (Tabla 3).

Tabla 2 - Estado nutricional de las gestantes ${ }^{(15)}$, según trimestre gestacional, en análisis longitudinal. Santo André, 1997

\section{Estado nutricional}

\begin{tabular}{|c|c|c|c|c|}
\hline $\begin{array}{l}\text { Trimestre } \\
\text { gestacional }\end{array}$ & $\begin{array}{c}\text { Bajo peso } \\
\text { ก (\$) }\end{array}$ & $\begin{array}{c}\text { Peso normal } \\
n(\infty)\end{array}$ & $\begin{array}{c}\text { Sobrepeso } \\
n(\%)\end{array}$ & $\begin{array}{l}\text { TOTÄL } \\
n(\mathscr{B})\end{array}$ \\
\hline $\begin{array}{l}1^{\text {er }} \text { Trimestre } \\
\text { ( }<13 \text { semanas) }\end{array}$ & $25(21,4)$ & $69(59,0)$ & $23(19,6)$ & $117(100,0)$ \\
\hline $\begin{array}{l}2^{\text {do }} \text { Trimestre } \\
\text { (14-28 semanas) }\end{array}$ & $24(20,5)$ & $68(58,1)$ & $25(21,4)$ & $117(100,0)$ \\
\hline $\begin{array}{l}3^{\text {er }} \text { Trimestre } \\
(\geq 29 \text { semanas })\end{array}$ & $22(18,8)$ & $62(53,0)$ & $33(28,2)$ & $117(100,0)$ \\
\hline
\end{tabular}

En Brasil, son escasos los estudios que evaluan prospectivamente el estado nutricional de la gestante durante el embarazo. Analizándose longitudinalmente los datos, no se verificó diferencia significativa entre las categorías de ubicación del estado nutricional, bajo peso, peso normal o sobrepeso, o sea, prácticamente todas las embarazadas que iniciaron la gestación con bajo peso permanecieron en esa condición hasta el final y algunas mujeres ubicadas con peso normal en el primer trimestre pasaron a la categoría de sobrepeso al tercer trimestre (Tabla 2).

Tabla 3 - Estado nutricional de las gestantes ${ }^{(15)}$, según edad gestacional, en análisis transversal. Santo André, 1997

\section{Estado nutricional}

\begin{tabular}{|c|c|c|c|c|}
\hline $\begin{array}{l}\text { Edad gestacional } \\
\text { (semanas) }\end{array}$ & $\begin{array}{c}\text { Bajo peso } \\
\text { ก (\$) }\end{array}$ & $\begin{array}{c}\text { Peso normal } \\
n(\%)\end{array}$ & $\begin{array}{c}\text { Sobrepeso } \\
n(\%)\end{array}$ & $\begin{array}{l}\text { TOTALL } \\
n(\%)\end{array}$ \\
\hline $10-13$ & $43(27,7)$ & $86(55,5)$ & $26(16,8)$ & $153(100,0)$ \\
\hline $14-20$ & $79(30,5)$ & $143(55,2)$ & $37(14,3)$ & $129(100,0)$ \\
\hline $21-28$ & $61(25,2)$ & $130(50,7)$ & $51(21,1)$ & $242(100,0)$ \\
\hline $29-33$ & $66(26,8)$ & $120(48,8)$ & $60(24,4)$ & $246(100,0)$ \\
\hline $34-40$ & $66(32,4)$ & $83(40,7)$ & $55(27,0)$ & $204(100,0)$ \\
\hline
\end{tabular}

* Chi cuadrado entre sobrepeso y bajo peso más peso normal $=14,88(P$ $=0,005$ )
El análisis transversal de los datos, que involucró la totalidad de las gestantes, mostró a su vez, una proporción mayor de mujeres ubicadas como de bajo peso $(25,2 \%$ a $32,4 \%)$ durante todo el embarazo. No obstante esa proporción de bajo peso ser elevada, ella fue similar a la prevalencia relatada en estudio con 1150 mujeres en el sur del país ${ }^{(27)}$, pero inferior a la proporción descrita al analizar algunos estudios aislados desarrollados en nuestro país ${ }^{(28)}$, y a los $40,7 \%$ anteriormente encontrados en embarazadas de bajo ingreso económico ${ }^{(26)}$. Apesar de ello, esta situación es preocupante, una vez que la manutención de la desnutrición durante el embarazo ha sido asociada con mayor incidencia de bajo peso al nacer ${ }^{(1)}$. Fue demonstrado que la incidencia de bajo peso al nacer era significativamente mayor (2,8 veces) entre las gestantes que no se recuperaron durante el embarazo, cuando comparadas con aquellas que se repecuperaron ${ }^{(5)}$.

Por otro lado, la proporción de gestantes ubicadas con sobrepeso aumentó, significativamente a medida que transcurrió la gestación, involucrando a más de un cuarto de las embarazadas al final de la gravidez, en detrimento de la proporción de mujeres de la categoria normal (Tabla 2 y 3). El valor encontrado es importante, una vez que el sobrepeso en la gestación, ha sido asociada significativamente con aumento de las taxas de morbi-mortalidad materna y perinatal.

La Tabla 4 muestra el estado nutricional materno durante el embarazo y el promedio del peso al nacer. Se verificó que el peso del infante al nacer tiende a aumentar conforme fue mejor ubicada nutricionalmente la madre en los tres trimestres gestacionales, sin embargo, diferencia significativa entre el estado nutricional de la madre y peso al nacer fue observada solamente a nivel del segundo trimestre.

Tabla 4 - Peso al nacer según estado nutricional ${ }^{(15)}$, según trimestre gestacional. Santo André, 1997

$\begin{array}{lccc}\text { Estado nutricional } & n & \text { Peso al nacer }(g) \times \pm d s & p \\ 1^{\text {er }} \text { Trimestre } & & & \\ \text { Bajo peso } & 12 & 3103 \pm 356 & \\ \text { Peso normal } & 27 & 3123 \pm 496 & 0,153 \\ \text { Sobrepeso } & 9 & 3541 \pm 964 & \\ & & & \\ 2^{\text {do }} \text { Trimestre } & & & \\ \text { Bajo peso } & 27 & 2966 \pm 447 & 0,012 \\ \text { Peso normal } & 50 & 3192 \pm 522 & \\ \text { Sobrepeso } & 15 & 3492 \pm 738 & \\ 3^{\text {er Trimestre }} & & & \\ \text { Bajo peso } & 30 & 3024 \pm 473 & \\ \text { Peso normal } & 42 & 3128 \pm 533 & 0,132 \\ \text { Sobrepeso } & 19 & 3494 \pm 672 & \end{array}$


Estudiando la desnutrición materna y bajo peso al nacer fue señalado que el riesgo de bajo peso se modifica en función de la evolución del estado nutricional durante el embarazo, pues cuando la desnutrición era corregida hasta el final del proceso, el riesgo de bajo peso al nacer tendía a disminuir a valores semejantes al de mujeres que mantuvieron normalidad de peso corporal durante toda la gestación ${ }^{(5)}$.

Tal como en el presente estudio, considerando la tendencia de las gestantes mantenerse en la categoría nutricional que inicia, esto es, bajo peso o sobrepeso, fue resaltado que el control prenatal no debe solamente buscar la prevención de la morbi-mortalidad perinatal o controlar las patologias de la gestación, pero también debe diagnosticar y monitorear el estado nutricional ${ }^{(29)}$.

Como las deficiencias nutricionales son socialmente determinadas, la erradicación completa del problema de la desnutrición depende de la propia erradicación de los grandes contrastes económicos y sociales generados y mantenidos por el proceso de producción y distribución de los bienes y servicios ${ }^{(30)}$.

Programas específicos del sector de salud pueden contribuir para un declínio substancial en la ocurrencia de las

\section{REFERENCIAS BIBLIOGRAFICAS}

1. Krasovec K, Anderson MA, editoras. Nutrición materna y resultados del embarazo: evaluación antropométrica. Washington (DC): Organización Panamericana de la Salud/Organización Mundial de la Salud; 1991. Publ Cient., nº 529.

2. Jelliffe DB. Evaluación del estado de nutrición de la comunidad (con especial refencia a las encuestas en las regiones de desarrollo). Ginebra (SZ), Organización Mundial de la Salud; 1968. (Serie de Monografía, $n^{0}$ 53).

3. Lechtig A, Delgado H, Lasky RE, Klein RE, Engle PL et al. Maternal nutrition and fetal growth in developing societes. Am J Dis Child 1975;129:434-7.

4. Barros FC, Victora CG, Vaughan JP, Estanislau HJ. Bajo peso al nacer en el Municipio de Pelotas, Brasil: factores de riesgo. Bol Of Sanit Panam 1987;120:541-53.

5. Sinisterra OT, Szarfarc SC, Benício MHD'A. Anemia e desnutrição maternas e sua relação com o peso ao nascer. Rev Saúde Pública 1991;25:193-7.

6. Alvarenga AT. Risco gravídico e risco perinatal: implicações metodológicas, teóricas e técnicas das propostas de mensuração. Rev Bras Cresc Des Hum 1994;4(2):41-8.

7. Ricalde $A E$, Siqueira AAF. El perímetro braquial en la mujer gestante, y su relación con otras medidas antropométricas maternas. Rev Bras Cresc Des Hum 1996;6(1/2):63-8. deficiencias nutricionales y, a corto plazo, medidas paliativas como la distribución de suplementos alimentares para mujeres en el último trimestre de la gestación, ayudan a corregir los casos de bajo peso en embarazadas ${ }^{(31)}$.

Así, concomitantemente, el control antropométrico adecuado, posibilita monitorear nutricionalmente a las embarazadas, previniendo y/o controlando la ocurrencia de condiciones maternofetales indeseables, hecho que reafirma la importancia de la ejecución de esta actividad como rutina en todo el control prenatal.

\section{AGRADECIMIENTOS}

Agradecemos la colaboración de los técnicos de la Fundación de Asistencia a la Infancia de Santo André, especialmente su coordenadora Alice Lang Simões Santos y subcoordenadora Iria Ramos. Este trabajo fue parcialmente financiado por la Fundación de Amparo a la Pesquisa del Estado de São Paulo - FAPESP ( $n^{\circ}$ 97/ 11849-2).

8. Garn SM, Pesick S. Relationship between various maternal body mass measures and size of the newborn. Am J Clin Nutr 1982;36:6648.

9. Martorell R. Talla materna como indicador de riesgo. In: Krasovec $\mathrm{K}$, Anderson MA, editors. Nutrición materna y resultados del embarazo: evaluación antropométrica. Washington (DC): Organización Panamericana de la Salud/Organización Mundial de la Salud; 1991. Publ Cient., nº 529.

10. Haas JD. Aumento de peso en la gestación. In: Krasovec K, Anderson MA, editors. Nutrición materna y resultados del embarazo: evaluación antropométrica. Washington (DC): Organización Panamericana de la Salud; 1991. Publ Cient., nº 529, 1991. Cap.2, p.33-58.

11. Ministério da Saúde (BR). Assistência integral à saúde da mulher: bases de ação programática. Brasília (DF): Ministério da Saúde, Centro de Documentação do Ministério da Saúde; 1984. Série B: Textos básicos de saúde 7 .

12. Garrow JS. Indices of adiposity. Nutr Abstr Rev 1983;53:697708.

13. Bray GA. Pathophysiology of obesity. Am J Clin Nutr 1992;55 Suppl 2:488-95.

14. World Health Organization. Diet, nutrition and prevention of chronic diseases. Geneva: WHO; 1990. Technical Report Series, $n^{0}$ 797.

15. Rosso P. A new chart to monitor gain during pregnancy. Am I Clin Nutr 1985;41:52-6. 
16. Tadei JAAC, Jacomo JÁ, Alburquerque ZP, Benício MHD'A, Athias

SP, Torres IHB et al. 0 percentual do peso para altura da gestante no prognóstico do baixo peso ao nascer: estudo multicêntrico. Rev Bras Ginecol Obstet 1991;2:78-82.

17. Ciari-Junior C, Almeida PAM, Siqueira AAF. Relação entre peso da criança ao nascer, altura materna, idade gestacional e restrição alimentar em gestantes normais. Rev Saúde Pública 1975;9:33-42. 18. Victora CG, Barros FC, Vaughan JP. Epidemiologia da desigualdade. São Paulo (SP): Hucitec; 1988.

19. Tomasi E, Barros FC, Victora CCG. Situação sócio-econômica e condições de vida: comparação de duas coortes de base populacional no sul do Brasil. Cad Saúde Pública 1996;12 Suppl 1:15-9.

20. Instituto Nacional de Alimentação e Nutrição (BR). Pesquisa nacional sobre saúde e nutrição: Perfil de crescimento da população brasileira de 0 a 25 anos. Brasília (DF): Instituto Nacional de Alimentação e Nutrição; 1990.

21. Benício MHD’A, Monteiro CA, Souza JMP, Castilho EA, Lamonica IMR. Análise multivariada de fatores de risco de baixo peso ao nascer em nascidos vivos - Município de São Paulo. Rev Saúde Pública 1985; 19:311-20.

22. Benício MHD'A. Fatores de risco de baixo peso ao nascer em recém-nascidos vivos: município de São Paulo, 1978. [tese]. São Paulo (SP): Faculdade de Saúde Pública/USP; 1983.

23. Lima CMG, Pelá NTR. Estudo com gestantes atendidas em um Centro Municipal de Saúde da periferia de Botucatu - São Paulo Relação entre baixo peso materno e o baixo peso ao nascer do concepto. Rev Latino-am Enfermagem 1995;3(1):79-94.
24. Sichieri R, Coitinho DC, Leão MM, Recine E, Everhart JE. High temporal, geographic and income variation in Body Mass Index amng adults in Brazil. Am J Publ Health 1994;84(5):793-8.

25. Fujimori E, Oliveira IMV, Nuñez de Cassana LM, Szarfarc SC. Estado nutricional de gestantes adolescentes en São Paulo, Brasil. Arch Latinoame Nutr 1997;47(4):305-10.

26. Suzuki E, Fujimori E, Nuñez de Cassana LM, Oliveira IMV. Perfil nutricional de la embarazada de bajo ingreso economico. São Paulo (SP), Brasil. Enf. Cient. 1997; (184-5):80-4.

27. Soares L, Más L, Cuozzo AM, Napp LM, Cunha AHM, Toniolo CE et al. Avaliação do estado nutricional na gestação. R C Matern Inf Ginecol 1990;9(2):28-35.

28. Castro IRR. Vigilância alimentar e nutricional: limitações e interfaces com a rede de saúde. Rio de Janeiro (RJ): Fiocruz; 1995. 29. Siqueira AAF, Ciari C Junior, Almeida PAM, Tanaka ACD'A, Montelone PPR, Arruda JJG, Delascio, D. Influência da altura e ganho de peso maternos e da idade gestacional sobre o peso do recém-nascido: estudo de 3 grupos de gestantes normais. Rev Saúde Pública 1975;9:331-42.

30. Hoffmann R. Pobreza, insegurança alimentar e desnutrição no Brasil. In: Seminário: pobreza, fome e desnutrição no Brasil, Instituto de Estudos Avançados da Universidade de São Paulo, São Paulo, 1994.

31. Batista-Filho M, Rissin A. Deficiências nutricionais: ações específicas do setor saúde para o seu controle. Cad Saúde Pública 1993; 9(2):130-5. 